\title{
Ankle complex musculature adaptations after Energy Drinks consumption in healthy young adult
}

\section{Martín G. Rosario PT, PhD, CSFI, ATRIC* (iD), Clare Hanrahan SPT (iD, Lauren Basye SPT (iD) and Ashley Correa SPT (D)}

a Texas Woman's University, School of Physical Therapy, Dallas, Texas

*Corresponding author Ph: (214) 689-7712 ; Email: mrosario1@twu.edu

DOI: https://doi.org/10.34256/ijpefs2124

Received: 15-03-2021, Revised: 12-04-2021; Accepted: 16-04-2021; Published: 27-04-2021

Abstract: Over the past few years, energy drink consumption has increased among students aged 18-34 years. Energy drinks alter the balance, reduce blood flow and interfere with neuromuscular activation in the lower extremities. We attempted to determine which specific additive of three different drinks (red bull, rockstar, and bang) could contribute to changes in muscle activation of the ankle complex. Twenty healthy young adults aged 22-28 years were included in this study and allocated among 3 groups, red bull, rockstar, and bang. Neuromuscular data were obtained from EMG sensors positioned on the anterior tibialis and gastrocnemius before completing the four balance tasks. Each participant completed all the tasks before and after the 16-ounce Edrink. ANOVA was performed to compare the data before and after the beverage. Statistical significance was set at $P<0.05$. A trend was observed in red bull and bang groups revealing a faster anterior tibial activity and prolong activation for gastrocnemius. The rockstar group also showed a more rapid activation trend and shorter response during all tasks for the gastrocnemius. It appears that the additives found in Redbull and Bang produce an increased posterior sway, indicated by the faster activity observed on the anterior tibial muscle. On the other hand, the elements encountered in rockstars provoke anterior movements, creating the need for a quicker response from the gastrocnemius muscle. Further research is required to explore certain energy drink ingredients' effects on dynamic activities such as walking.

Keywords: Energy drinks, Muscle activation, Tibialis anterior, Gastrocnemius, Energy drink ankle musculature
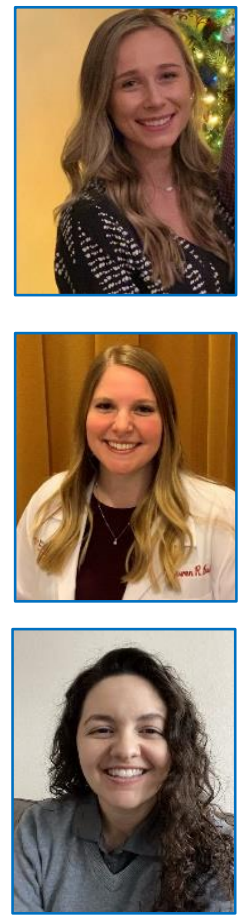

Clare Hanrahan, SPT. Will obtain her Doctor of Physical Therapy (DPT) degree from Texas Woman's University-Dallas in May 2021. Obtained her Bachelor of Science in Kinesiology from Texas Christian University.

Lauren Basye, SPT. Will obtain her Doctor of Physical Therapy (DPT) degree from Texas Woman's University-Dallas in May 2021. Obtained her Bachelor of Science in Psychology from Texas A\&M University.

Ashley V. Correa, SPT. Will obtain her DPT from Texas Woman's University in May 2021. Bachelor in Business Management with an emphasis in Organizational Behavior and Human Resources from Brigham Young University.

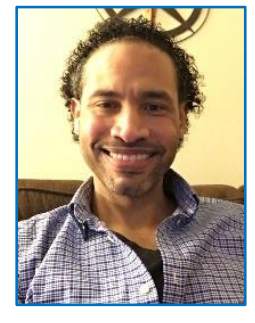

Martin G. Rosario PT, Ph.D., CSFI, ATRIC. Obtained his Ph.D. in Neuroanatomy from the University of Puerto Rico Medical Sciences Campus. Master in Physical Therapy from the University of Puerto Rico Medical Sciences Campus School of Physical Therapy. Bachelor in Biology with a Major in Human Focus from University of Puerto Rico Bayamon Campus. Currently serves as an Assistant Professor at Texas Woman's University (TWU) School of Physical Therapy Dallas, Clinical Researcher at an HIV community clinic La Perla de Gran Precio in Puerto Rico, and Director/Coordinator of Motor Control Research Laboratory at TWU Dallas.

Int. J. Phys. Educ. Fit. Sports, 10(2) (2021), 32-44 | 32 


\section{Introduction}

The consumption of energy drinks (Edrinks) gained popularity as sales increased by more than double between 2004 and 2009 [1]. Based on an inquiry by Burrows et al., [1], young male adults in college are among the primary consumers of Edrinks. Most of the attention has been paid to advertising the advantages of Edrinks. Among the declared benefits, Edrinks are reported to promote athletic performance, concentration, response time, basal metabolic rate, and increase energy levels [1].

The problem is that Edrinks are labeled as a supplement and therefore not regulated in the US [1], generating further prominent issues by the use and misuse of this beverage. Depending on the brand, Edrinks vary depending on the ingredients and the number of additives declared by the manufacturer. What is troubling is that some of these elements have their own risk. For example, among the essential ingredients in Edrinks, the most prevalent is caffeine, which is found to increase alertness and mood and neutralize the effects of sleep loss. Nevertheless, it is noted that caffeine causes gastrointestinal distress, nausea, insomnia, and withdrawal symptoms, such as headaches. Caffeine toxicity is a possibility with Edrinks utilization, which is why the FDA has imposed a limit of $71 \mathrm{mg}$ of caffeine per 12 fluid ounces of soda [2]. However, Edrinks companies seek to push back these regulations by advertising their products as "natural dietary supplements" [2].

Visits to emergency services due to the use of Edrinks doubled in 2007-2011 and have since increased in the United States [3]. What is worrying is that apart from caffeine, other elements such as taurine, guarana, glucuronolactone, ginseng, and L-carnitine are incorporated into the mixture of most of this drink. Most people are aware of caffeine intake because the amount is stated on the label; however, many other ingredients are largely unknown [3].

Cardiovascular complications, such as lifethreatening heart rate anomalies, are another problem associated with Edrinks. Edrinks, such as RockStar, have also been reported to increase blood pressure and the level of stress hormones released by the adrenal gland in the blood, which are risk factors for cardiovascular events [4]. Other cardiovascular side effects include increased diastolic blood pressure, heart palpitations, and platelet aggregation [5]. Couple with the influence of ingredients such as carnitine, which is linked to atherosclerosis, many of these drinks can trigger endothelial dysfunction, leading to a blood flow reduction [6]. A decrease in blood flow [7] to peripheral structures, including the muscles and nerves of the extremities, could increase fatigue, thereby modifying standing balance.

Few studies have examined the connection between Edrinks and caffeine and the central nervous system (CNS) [8-13]. Caffeine by itself has the potential to stimulate the central nervous system [12] and leads to an increase in sway in healthy young adults after a 200-milligram pill [14]. Combined with caffeine, Edrinks have been associated with the development of dependency risk, increased impulsivity, and reduced academic achievement [15]. Furthermore, changes in lower limb muscle activation patterns during standing tasks among college students [16].

As noted above, Edrinks often incorporate diverse elements such as taurine, guarana, and riboflavin, which can modify the central nervous system (CNS) [17]. There is no question that many of these additives have unique properties, and the lack of information relates to the failure to adequately address the consequences of mixing these ingredients when they are collectively ingested [6]. Concerning all the above, we strive to determine the impact of 3 distinct energetic drinks with unique components on balance and muscle activation during multitasking activities. Standing balance is influenced by the CNS, and the synergy of these three major systems achieves motor control (visual, somatosensory, and vestibular) [18]. Therefore, it is imperative to distinguish the association between caffeine and drink ingredients on the balance and muscular activation of the lower limbs.

The query under consideration is whether energy drinks or a distinct ingredient can alter muscle activation and balance in healthy young adults. We assume that Edrinks will lead to modifications in the ankle complex musculature, such as a faster onset and shorter muscle activity duration. This inquiry will add to the gap in Edrinks' unknown effects on muscle activity and postural control that are likely to affect Edrink users.

\section{Methods}

We included 20 healthy adults, 2 men, and 18 women. Ages varied from 22 to 28 years old, with an average age of 23.9 years old ( $+/-1.85$ years, Table 1$)$, and all participants were Texas Woman's University graduates enrolled in Physical Therapy, Occupational Therapy, or Nursing programs. The exclusion criteria consisted of a history of diabetes, heart problems, and 
back or leg injuries six months before the examination. After signing the informed consent and before consuming the energy drink, participants were asked about their dominant leg in which the electromyography (EMG) sensors were located as previously illustrated by Rosario et al., [16].

In this study were collected neuromuscular activity using an EMG surface electrode system (Delsys, Inc. Boston, MA) on the tibialis anterior (TA) and gastrocnemius (GA) muscles, and the activities of the two muscles were acquired at $1,000 \mathrm{~Hz}$. This two muscles, TA and GA are major players in ankle strategies when performing standing balance activities. Subsequently, participants found themselves on a pressure platform where the COP data were collected along with the EMG data. For the balance protocol, participants were initially asked to stand for $15 \mathrm{~s}$ while holding a full water cup in their dominant hand. During all open-eye tasks, participants were asked to focus on an area marked on the wall at the eye level. The participants were expected to stand on foam to recreate an unstable surface and challenge the proprioceptive system for all tasks. To challenge the vestibular input, participants moved their head up and down, flexed, and extended in a rhythmic technique with a speed of 60 beats per minute (bpm). We asked the participants to find and keep up the pace during the nodding tasks before starting the test. EMG sensors simultaneously recorded the muscular activity of the leg muscles (anterior tibialis and gastrocnemius).

The distinct sequence for the trial was as follows:

(a) Standing on an unstable foam surface with eyes open, focusing on a marked spot on the wall at the eye level. b) Standing on the (unstable) foam surface with eyes closed; c) Place on a foam surface (unstable) with eyes open while tilting the head up and down at 60 bpm (look remains on a mark on the wall) d) continue on a foam surface (unstable), tilting head upwards and downwards at $60 \mathrm{bpm}$ with eyes closed.

Afterward, participants drank the 16-ounce energy drink (Rockstar, Red Bull, or Bang) at their own pace. Therefore, to detect cardiovascular changes, such as an increase in heart rate by ten beats per minute, balance protocols were repeated to compare data for pre-energy drinks and post-energy drinks.
Data Analysis

Version 25 of the Statistical Package for the Social Sciences (SPSS) was used for all data analyses. A MANOVA with posthoc comparisons was conducted with all the variables of interest between pre-and postenergy drink consumption. EMG muscle activation data will include 1) duration (secs), 2) time to a maximum peak of activation (secs), and 3) time to decay maximum activation (secs). $P$ values equal to or less than 0.05 will be accepted as statistically significant. Gathering this information will help determine the impact of energy drinks on balance and muscle activity.

\section{Results}

Table 1 presents the demographic characteristics of the participants, including age, sex, and BMI. The demographics of the Redbull, Bang, and Rockstar groups were comparable among the three groups. The results of the participants' cardiovascular data are shown in Table 2. For the Redbull group, the average blood pressure, heart rate, and oxygen saturation increased post-EDC. In the Bang group, mean blood pressure also increased after EDC; however, mean heart rate and oxygen saturation declined after EDC. With Rockstar, the mean blood pressure and heart rate increased after EDC, and oxygen saturation decreased slightly. To summarize, the average blood pressure increased in all three groups after $C D E$, while heart rate and oxygen saturation varied.

Tables 3, 5, 7, and 9 reveal the outcomes of neuromuscular activity for the TA muscle, and Tables 4 , 6,8 , and 10 depict the findings of the neuromuscular activity for GA muscle among the various balance activities. While the data were similar across groups, after consuming energy drinks, the present study shows a similar propensity for activation of the Red Bull and Bang groups' ankle muscles. However, a reverse trend was observed in the rock star cohort. Red bulls and bang induce a quicker onset of activity; however, rockstars generate a TA's delayed activation response. In contrast, during the same tasks, red bulls and bang produced delayed onset and prolonged activation for the GA muscle. At the same time, the rockstar group showed tendencies of faster activation and shorter reaction during all tasks for the GA muscle. 
Table 1 Characteristics of the study participants

\begin{tabular}{|l|l|l|l|}
\hline Characteristics & Redbull $(\mathrm{n}=6)$ & Bang $(\mathrm{n}=7)$ & Rockstar $(\mathrm{n}=7)$ \\
\hline Age & $\mathrm{m}=23.5+/-0.5$ & $\mathrm{~m}=24.5+/-2.6$ & $\mathrm{~m}=23.7+/-1.9$ \\
\hline Male & $\mathrm{n}=1$ & $\mathrm{n}=0$ & $\mathrm{n}=1$ \\
\hline Female & $\mathrm{n}=5$ & $\mathrm{n}=7$ & $\mathrm{n}=6$ \\
\hline Height-inches & $\mathrm{m}=68.1+/-4.4$ & $\mathrm{~m}=66.8+/-2.8$ & $\mathrm{~m}=66.7+/-3.1$ \\
\hline Weight-pounds & $\mathrm{m}=157.4+/-36.6$ & $\mathrm{~m}=135.3+/-6.6$ & $\mathrm{~m}=152.7+/-23.1$ \\
\hline
\end{tabular}

Table 2 Characteristics of participants cardiovascular Data

\begin{tabular}{|l|l|l|}
\hline Redbull & Pre Edrink & Post Edrink \\
\hline Systolic (mmHg) & $112.8+/-10.87$ & $117.6+/-18.64$ \\
\hline Diastolic (mmHg) & $74.4+/-6.46$ & $78.8+/-5.80$ \\
\hline Heart Rate (beats per minute) & $70.4+/-5.17$ & $80+/-8.80$ \\
\hline Oxygen Saturation (\%) & $97.6+/-1.51$ & $98+/-1.41$ \\
\hline Time for Cardio Change (minutes) & & $11.8+/-5.44$ \\
\hline Bang & Pre Edrink & Post Edrink \\
\hline Systolic (mmHg) & $113.14+/-8.13$ & $114.85+/-7.42$ \\
\hline Diastolic (mmHg) & $78.14+/-6.44$ & $83.28+/-9.70$ \\
\hline Heart Rate (beats per minute) & $73+/-17.27$ & $70.28+/-11.27$ \\
\hline Oxygen Saturation (\%) & $98+/-1$ & $97.14+/-2.26$ \\
\hline Time for Cardio Change (minutes) & & $13.71+/-3.14$ \\
\hline Rockstar & Pre Edrink & Post Edrink \\
\hline Systolic (mmHg) & $117+/-11.81$ & $121.14+/-12.95$ \\
\hline
\end{tabular}




\begin{tabular}{|l|l|l|}
\hline Diastolic (mmHg) & $75.14+/-3.93$ & $87.14+/-6.03$ \\
\hline Heart Rate (beats per minute) & $73.71+/-15.15$ & $78.85+/-18.95$ \\
\hline Oxygen Saturation (\%) & $96.57+/-3.35$ & $96.42+/-2.37$ \\
\hline Time for Cardio Change (minutes) & & $13+/-2.44$ \\
\hline
\end{tabular}

Table 3 EMG data for TA muscle during EO task. Results of repeated measure ANOVA between pre-energy drink and post-energy drink. Significance level set at $p \leq 0.05$.

\begin{tabular}{|c|c|c|c|}
\hline Redbull & Pre Edrink & Post Edrink & $P$ value \\
\hline onset & $10.2067+/-4.1443$ & $7.4683+/-3.8796$ & 0.26 \\
\hline Time to peak & $0.2700+/-0.2690$ & $0.2983+/-0.1461$ & 0.86 \\
\hline duration & $0.6967+/-0.6044$ & $0.6683+/-0.1824$ & 0.67 \\
\hline decay & $0.4267+/-0.3422$ & $0.3700+/-0.2264$ & 0.91 \\
\hline Bang & Pre Edrink & Post Edrink & $P$ value \\
\hline onset & $8.0429+/-4.5154$ & $7.7586+/-5.0515$ & 0.90 \\
\hline Time to peak & $0.2071+/-0.1413$ & $0.4729+/-0.5120$ & 0.48 \\
\hline duration & $0.5814+/-0.3541$ & $0.8471+/-0.6765$ & 0.05 \\
\hline decay & $0.3743+/-0.2229$ & $0.3743+/-0.1729$ & 0.24 \\
\hline Rockstar & Pre Edrink & Post Edrink & $P$ value \\
\hline onset & $8.3386+/-3.6189$ & $12.4971+/-3.3425$ & 0.07 \\
\hline Time to peak & $0.1871+/-0.0626$ & $0.2814+/-0.2149$ & 0.33 \\
\hline duration & $0.4029+/-0.1106$ & $0.6143+/-0.3805$ & 0.20 \\
\hline decay & $0.2157+/-0.1052$ & $0.3329+/-0.2442$ & 0.13 \\
\hline
\end{tabular}


Table 4 EMG data for gastroc muscle during EO task. Results of repeated measure ANOVA between pre-energy drink and post-energy drink. Significance level set at $p \leq 0.05$.

\begin{tabular}{|l|l|l|l|}
\hline Redbull & Pre Edrink & Post Edrink & P value \\
\hline onset & $8.7233+/-3.8517$ & $9.0633+/-4.3769$ & 0.88 \\
\hline Time to peak & $0.4150+/-0.2269$ & $0.4567+/-0.3681$ & 0.76 \\
\hline duration & $0.7483+/-0.3354$ & $0.6967+/-0.4857$ & 0.77 \\
\hline decay & $0.3333+/-0.2507$ & $0.2400+/-0.1585$ & 0.32 \\
\hline Bang & Pre Edrink & Post Edrink & $P$ value \\
\hline onset & $7.1971+/-4.2639$ & $11.7986+/-3.9220$ & 0.05 \\
\hline Time to peak & $0.4100+/-0.1422$ & $0.3700+/-0.1901$ & 0.75 \\
\hline duration & $0.6500+/-0.1545$ & $0.7643+/-0.2931$ & 0.47 \\
\hline decay & $0.2400+/-0.1111$ & $0.3943+/-0.2043$ & 0.08 \\
\hline Rockstar & Pre Edrink & Post Edrink & $P$ value \\
\hline onset & $9.6500+/-4.0394$ & $7.7057+/-2.9979$ & 0.26 \\
\hline Time to peak & $0.2657+/-0.1713$ & $0.3757+/-0.2465$ & 0.34 \\
\hline duration & $0.4671+/-0.2120$ & $0.5971+/-0.2347$ & 0.62 \\
\hline decay & $0.2014+/-0.0890$ & $0.2214+/-0.0890$ & 0.82 \\
\hline Units for onset, time to peak, duration and decay in seconds. & \\
\hline & & & \\
\hline
\end{tabular}

Table 5 EMG data for TA muscle during EC task. Results of repeated measure ANOVA between pre-energy drink and post-energy drink. Significance level set at $p \leq 0.05$

\begin{tabular}{|l|l|l|l|}
\hline Redbull & Pre Edrink & Post Edrink & P value \\
\hline onset & $8.8450+/-4.36102$ & $9.1067+/-3.04484$ & 0.09 \\
\hline Time to peak & $0.1900+/-0.05367$ & $0.2233+/-0.14264$ & 0.72 \\
\hline
\end{tabular}




\begin{tabular}{|l|l|l|l|}
\hline duration & $0.3983+/-0.11548$ & $0.4483+/-0.16388$ & 0.92 \\
\hline decay & $0.2083+/-0.09988$ & $0.2250+/-0.08643$ & 0.89 \\
\hline Bang & Pre Edrink & Post Edrink & P value \\
\hline onset & $6.6586+/-2.76529$ & $8.5100+/-5.12155$ & 0.34 \\
\hline Time to peak & $0.4243+/-0.40566$ & $0.5643+/-0.63232$ & 0.68 \\
\hline duration & $0.8457+/-0.54212$ & $1.0257+/-0.89976$ & 0.20 \\
\hline decay & $0.4214+/-0.19256$ & $0.4614+/-0.30019$ & 0.73 \\
\hline Rockstar & Pre Edrink & Post Edrink & $P$ value \\
\hline onset & $6.0500+/-1.94447$ & $5.4557+/-3.41611$ & 0.76 \\
\hline Time to peak & $0.2600+/-0.12702$ & $0.7814+/-1.30765$ & 0.14 \\
\hline duration & $0.4029+/-0.1106$ & $0.6143+/-0.3805$ & 0.45 \\
\hline decay & $0.2843+/-0.11928$ & $0.3800+/-0.31485$ & 0.40 \\
\hline Units for onset, time to peak, duration and decay in seconds. & \\
\hline
\end{tabular}

Table 6 EMG data for gastroc muscle during EC task. Results of repeated measure ANOVA between pre-energy drink and post-energy drink. Significance level set at $p \leq 0.05$

\begin{tabular}{|l|l|l|l|}
\hline Redbull & Pre Edrink & Post Edrink & P value \\
\hline onset & $9.1150+/-4.64330$ & $10.4250+/-3.87630$ & 0.56 \\
\hline Time to peak & $0.4250+/-0.14775$ & $0.4683+/-0.14593$ & 0.89 \\
\hline duration & $0.8450+/-0.31072$ & $0.9367+/-0.38842$ & 0.85 \\
\hline decay & $0.4200+/-0.19860$ & $0.4683+/-0.27177$ & 0.82 \\
\hline Bang & Pre Edrink & Post Edrink & $P$ value \\
\hline onset & $9.4100+/-3.29298$ & $7.1286+/-4.17814$ & 0.28 \\
\hline Time to peak & $0.4229+/-0.40173$ & $0.7500+/-0.59161$ & 0.27 \\
\hline
\end{tabular}




\begin{tabular}{|l|l|l|l|}
\hline duration & $0.9914+/-0.53714$ & $1.3529+/-0.82381$ & 0.43 \\
\hline decay & $0.5686+/-0.21474$ & $0.6029+/-0.28669$ & 0.86 \\
\hline Rockstar & Pre Edrink & Post Edrink & P value \\
\hline onset & $7.4371+/-3.70537$ & $10.6729+/-3.66968$ & 0.26 \\
\hline Time to peak & $0.2957+/-0.15757$ & $0.6571+/-1.07014$ & 0.23 \\
\hline duration & $0.6614+/-0.50706$ & $0.9243+/-1.59732$ & 0.56 \\
\hline decay & $0.3657+/-0.36792$ & $0.2671+/-0.63754$ & 0.62 \\
\hline
\end{tabular}

Units for onset, time to peak, duration and decay in seconds.

Table 7 EMG data for TA muscle during EOHUD task. Results of repeated measure ANOVA between pre-energy drink and post-energy drink. Significance level set at $p \leq 0.05$

\begin{tabular}{|l|l|l|l|}
\hline Redbull & Pre Edrink & Post Edrink & P value \\
\hline onset & $10.2067+/-4.1443$ & $7.4683+/-3.8796$ & 0.70 \\
\hline Time to peak & $0.2700+/-0.2690$ & $0.2983+/-0.1461$ & 0.52 \\
\hline duration & $0.6967+/-0.6044$ & $0.6683+/-0.1824$ & 0.37 \\
\hline decay & $0.4267+/-0.3422$ & $0.3700+/-0.2264$ & 0.62 \\
\hline Bang & Pre Edrink & Post Edrink & P value \\
\hline onset & $8.0429+/-4.5154$ & $7.7586+/-5.0515$ & 0.99 \\
\hline Time to peak & $0.2071+/-0.1413$ & $0.4729+/-0.5120$ & 0.81 \\
\hline duration & $0.5814+/-0.3541$ & $0.8471+/-0.6765$ & 0.59 \\
\hline decay & $0.3743+/-0.2229$ & $0.3743+/-0.1729$ & 0.55 \\
\hline Rockstar & Pre Edrink & Post Edrink & P value \\
\hline onset & $8.3386+/-3.6189$ & $12.4971+/-3.3425$ & 0.87 \\
\hline Time to peak & $0.1871+/-0.0626$ & $0.2814+/-0.2149$ & 0.45 \\
\hline
\end{tabular}




\begin{tabular}{|l|l|l|l|}
\hline duration & $0.4029+/-0.1106$ & $0.6143+/-0.3805$ & 0.76 \\
\hline decay & $0.2157+/-0.1052$ & $0.3329+/-0.2442$ & 0.89 \\
\hline
\end{tabular}

Units for onset, time to peak, duration and decay in seconds.

Table 8 EMG data for gastroc muscle during EOHUD task. Results of repeated measure ANOVA between pre-energy drink and post-energy drink. Significance level set at $p \leq 0.05$

\begin{tabular}{|l|c|c|c|}
\hline \multicolumn{1}{|c|}{ Redbull } & Pre Edrink & Post Edrink & P value \\
\hline onset & $8.7233+/-3.8517$ & $9.0633+/-4.3769$ & 0.92 \\
\hline Time to peak & $0.4150+/-0.2269$ & $0.4567+/-0.3681$ & 0.65 \\
\hline duration & $0.7483+/-0.3354$ & $0.6967+/-0.4857$ & 0.70 \\
\hline decay & $0.3333+/-0.2507$ & $0.2400+/-0.1585$ & 0.46 \\
\hline Bang & \multicolumn{1}{|c|}{ Pre Edrink } & Post Edrink & P value \\
\hline onset & $7.1971+/-4.2639$ & $11.7986+/-3.9220$ & 0.26 \\
\hline Time to peak & $0.4100+/-0.1422$ & $0.3700+/-0.1901$ & 0.8 \\
\hline duration & $0.6500+/-0.1545$ & $0.7643+/-0.2931$ & 0.6 \\
\hline decay & $0.2400+/-0.1111$ & $0.3943+/-0.2043$ & 0.50 \\
\hline Rockstar & Pre Edrink & Post Edrink & P value \\
\hline onset & $9.6500+/-4.0394$ & $7.7057+/-2.9979$ & 0.21 \\
\hline Time to peak & $0.2657+/-0.1713$ & $0.3757+/-0.2465$ & 0.54 \\
\hline duration & $0.4671+/-0.2120$ & $0.5971+/-0.2347$ & 0.67 \\
\hline decay & $0.2014+/-0.0890$ & $0.2214+/-0.0890$ & 0.99 \\
\hline Units for onset, time to peak, duration and decay in seconds. & \\
\hline & & & \\
\hline
\end{tabular}


Table 9 EMG data for TA muscle during ECHUD task. Results of repeated measure ANOVA between pre-energy drink and post-energy drink. Significance level set at $p \leq 0.05$.

\begin{tabular}{|l|l|l|l|}
\hline Redbull & Pre Edrink & Post Edrink & P value \\
\hline onset & $8.0150+/-4.48384$ & $6.8517+/-3.66867$ & 0.65 \\
\hline Time to peak & $0.2100+/-0.09716$ & $0.5667+/-0.56245$ & 0.32 \\
\hline duration & $0.4467+/-0.09438$ & $0.9367+/-0.70324$ & 0.35 \\
\hline decay & $0.2367+/-0.09480$ & $0.3700+/-0.22244$ & 0.51 \\
\hline Bang & Pre Edrink & Post Edrink & $P$ value \\
\hline onset & $8.7300+/-5.25478$ & $9.0943+/-4.23042$ & 0.88 \\
\hline Time to peak & $0.2357+/-0.13402$ & $0.3586+/-0.24647$ & 0.71 \\
\hline duration & $0.4129+/-0.31878$ & $0.8286+/-0.50946$ & 0.39 \\
\hline decay & $0.1771+/-0.30527$ & $0.4700+/-0.27592$ & 0.54 \\
\hline Rockstar & Pre Edrink & Post Edrink & P value \\
\hline onset & $9.4871+/-4.31619$ & $8.8100+/-3.85057$ & 0.77 \\
\hline Time to peak & $0.9257+/-1.31627$ & $0.2514+/-0.12589$ & $\mathbf{0}$ \\
\hline duration & $1.4586+/-1.90012$ & $0.5443+/-0.33291$ & 0.63 \\
\hline decay & $0.5329+/-0.60494$ & $0.2929+/-0.27439$ & 0.3 \\
\hline Units for onset, time to peak, duration and decay in seconds. & \\
\hline & & & \\
\hline
\end{tabular}

Table 10 EMG data for gastroc muscle during ECHUD task. Results of repeated measure ANOVA between pre-energy drink and post-energy drink. Significance level set at $p \leq 0.05$.

\begin{tabular}{|l|l|l|l|}
\hline Redbull & Pre Edrink & Post Edrink & P value \\
\hline onset & $11.4117+/-3.09267$ & $7.8267+/-3.06129$ & 0.82 \\
\hline Time to peak & $0.2800+/-0.20248$ & $0.3117+/-0.16327$ & 0.85 \\
\hline duration & $0.6650+/-0.47669$ & $0.7800+/-0.33799$ & 0.7 \\
\hline
\end{tabular}




\begin{tabular}{|l|l|l|l|}
\hline decay & $0.3850+/-0.29569$ & $0.4683+/-0.24457$ & 0.64 \\
\hline Bang & Pre Edrink & Post Edrink & P value \\
\hline onset & $6.2814+/-2.79988$ & $7.9529+/-3.34208$ & 0.62 \\
\hline Time to peak & $0.4271+/-0.48938$ & $0.4071+/-0.25786$ & 0.9 \\
\hline duration & $0.8586+/-0.67979$ & $0.9571+/-0.40302$ & 0.7 \\
\hline decay & $0.4314+/-0.21729$ & $0.5500+/-0.23573$ & 0.48 \\
\hline Rockstar & Pre Edrink & Post Edrink & $P$ value \\
\hline onset & $9.6100+/-5.46993$ & $6.4571+/-4.88274$ & 0.14 \\
\hline Time to peak & $0.3486+/-0.23061$ & $0.2700+/-0.26758$ & 0.62 \\
\hline duration & $0.5629+/-0.74184$ & $0.5129+/-0.32587$ & 0.86 \\
\hline decay & $0.2143+/-0.54723$ & $0.2429+/-0.12816$ & 0.87 \\
\hline Units for onset, time to peak, duration and decay in seconds. & \\
\hline
\end{tabular}

\section{Discussion}

This study aimed to investigate the impact of Edrinks on complex ankle muscles during several balancing activities among healthy young adults. We speculate that Edrinks or a combination of beverage ingredients alter the ankle complex muscle's activation strategy. The findings of this study highlight the distinct patterns of muscle activation in various beverages.

Considering the ankle joint postural balance strategy, we reviewed the patterns of these muscles in four distinct balancing tasks between three different Edrinks: Red Bull, Bang, and Rockstar. Postural control is performed by consolidating three central systems: visual, proprioceptive, and vestibular inputs. We designed various balance tasks in this investigation to defy visual information by closing the eyes and relying on the other two inputs. Standing on an unsteady surface challenges proprioceptive information and moves the head upward/downward to generate vestibular interaction. A combination of two or more of these tests was built to prompt and, to a certain extent, foresee the displacement of the new in-charge balance system, providing equilibrium. This earlier concept of switching importance to one system is known as sensory reweighting. Generally, sensory reweighting is continuously employed, and in a typical equilibrium system, the subject will never become aware of this interaction unless two or more of the networks are pathological. We expect that the formerly stated and the dual motor tasks, holding the water cup, will elicit a sensory overload in the balanced interplay that generally a healthy body will quickly adjust. However, in the presence of some ingredients encountered in Edrinks, new ankle strategies may emerge after Edrink.

After consuming energy drinks, the current study showed a similar propensity for muscle activation of the ankle muscles among all balance tasks. TA: Red Bull and bang trigger a quicker start to activity; however, rockstars generate a delayed activation response. In contrast, red bulls and bang yielded a delayed start and prolonged activation during the same tasks. GA: The rockstar group showed faster activation trends and shorter responses during all tasks.

The intricate ankle muscles, such as the GA and $T A$, have a unique postural balance control pattern. In the passive standing position, in both feet on the 
ground, the GA muscle is triggered by excessive anterior oscillation beyond the median line. These ankle movements and GA activation indicate that the ankle is in dorsiflexion rather than neutral [18], which is similar to the response displayed by the rockstar group. In contrast, the TA muscle engages once the rear sway is over the neutral position of the ankle. The body needs to be corrected anteriorly [18], parallel to the outcome demonstrated by the Bang and Redbull groups.

One probable reason for these findings might be that rockstars have more ingredients than Redbull and bang, such as ginkgo biloba and guarana. These two additives induce variations at a more physiological level. In addition to the caffeine in Edrinks, guarana is a plant containing four times the caffeine content in coffee bean [17]. Previous studies have shown how guarana can improve cognitive behavior, mental lethargy, and sports-practice mood at appropriate doses and promote lipid metabolism [19]. On the other hand, Ginkgo Biloba is shown for vasomotor properties and functions related to improved circulation [20].

Another reason for our results might be associated with the B-vitamins found in Bang and Redbull. B-vitamins (B9 folic acid and B12) are known for increase nerve conduction velocity. The Bang and Red Bull drinks used in our analysis contain B vitamins such as niacin, inositol, riboflavin, and B12, which explains the difference in the ankle muscle variation [2021]. To further the above position, Rosario et al., [16] identified comparable adjustments in ankle musculature neuromuscular patterns alluded to in their study, however, with a monster beverage. The Monster Energy Drink is known to contain B vitamins in a mixture of ingredients, suggesting that these components are related to the neuromuscular alterations detected in both studies.

\section{Conclusion}

The study investigated the influence of ingredients within Edrinks on the ankle complex musculature during several complex balance tasks. Ankle musculature, TA, exhibited a trend of faster muscle recruitment in most of the tasks in the groups that consumed the ingredients mentioned above, for bang and red bulls. On the other hand, the rock star group showed a delayed activity response for the same tasks. However, the opposite was observed for GA muscle. Given that this was an exploratory study to inform future research directions, we were unable to produce any definitive conclusions. Nevertheless, prospective considerations should delve into guarana, Ginkgo biloba, and B-complex vitamin impact on neuromuscular properties. These recommended forthcoming inquiries should inspect the individual and combined effects of the supplements on rapid reaction musculature, such as the ankle muscle complex, to further understand its role in muscle activation further. This study makes it possible to identify the impact of various elements of the Edrinks and their effects on muscular activation. Subsequent considerations should address the influence of this beverages under more demanding conditions, such as single-leg balance and dynamic activities such as walking.

\section{References}

[1] T. Burrows, K. Pursey, M. Neve, P. Stanwell, What are the health implications associated with the consumption of energy drinks? A systematic review, Nutrition Reviews, Volume 71(3) (2013) 135-148. [DOI] | [PubMed]

[2] V. De Sanctis, N. Soliman, A.T. Soliman, H. Elsedfy, S. Di Maio, M. El Kholy, B. Fiscina, Caffeinated energy drink consumption among adolescents and potential health consequences associated with their use: a significant public health hazard. Acta Biomedica, 88(2) (2017) 222231. [DOI] | [PubMed]

[3] B. Wassef, M. Kohansieh, A.N. Makaryus, Effects of energy drinks on the cardiovascular system, World Journal of Cardiology, 9(11) (2017) 796806. [DOI] | [PubMed]

[4] A. Svatikova, N. Covassin, K.R. Somers, K.V. Somers, F. Soucek, T. Kara, J. Bukartyk, A Randomized Trial of Cardiovascular Responses to Energy Drink Consumption in Healthy Adults, JAMA， 314(19) (2015) 2079-2082. [DOI] । [PubMed]

[5] F. Sanchis-Gomar, R. Leischik, \& G. Lippi, Energy drinks: Increasing evidence of negative cardiovascular effects, International Journal of Cardiology, 206, (2016) 153. [DOI] | [PubMed]

[6] G. Lippi, G. Cervellin, \& F. Sanchis-Gomar, Energy Drinks and Myocardial Ischemia: A Review of Case Reports, Cardiovascular Toxicology, 16(3) (2015) 207-212. [DOI] | [PubMed]

[7] J.P. Higgins, B. Yang, N.E. Herrin, S. Yarlagadda, G.T. Le, B.L. Ortiz, A. Ali, S.C. Infanger, Consumption of energy beverage is associated with attenuation of arterial endothelial flowmediated dilatation, World Journal of Cardiology, 9(2) (2017) 162-166. [DOI] | [PubMed]

[8] J.J. Breda, S.H. Whiting, R. Encarnação, S. Norberg, R. Jones, M. Reinap, J. Jewell, Energy Drink Consumption in Europe: A Review of the 
Risks, Adverse Health Effects, and Policy Options to Respond, Frontiers in Public Health, 2 (2014) 134. [DOI] | [PubMed]

[9] N.B. Collier, M.A. Hardy, M.L. Millard-Stafford, G.L. Warren, Small Beneficial Effect of Caffeinated Energy Drink Ingestion on Strength, Journal of Strength and Conditioning Research, 30(7) (2016) 1862-1870. [DOI] | [PubMed]

[10] E.A. Fletcher, C.S. Lacey, M. Aaron, M. Kolasa, A. Occiano, S.A. Shah, Randomized Controlled Trial of High-Volume Energy Drink Versus Caffeine Consumption on ECG and Hemodynamic Parameters Journal of the American Heart Association, 6(5) (2017) e004448. [DOI] । [PubMed]

[11] W.P. Mccormack, J.R. Hoffman, Caffeine, Energy Drinks, and Strength-Power Performance, Strength and Conditioning Journal, 34(4) (2012) 11-16. [DOI]

[12] D. Nowak, M. Gośliński, K. Nowatkowska, The Effect of Acute Consumption of Energy Drinks on Blood Pressure, Heart Rate and Blood Glucose in the Group of Young Adults, 15(3) (2018) 544. [DOI] | [PubMed]

[13] M.A. Trevino, J.W. Coburn, L.E. Brown, D.A. Judelson, M.H. Malek, Acute Effects of Caffeine on Strength and Muscle Activation of the Elbow Flexors, Journal of Strength and Conditioning Research, 29(2) (2015) 513-520. [DOI] | [PubMed]

[14] M.G. Rosario, H. Collazo, M. Mateo, M. GonzalezSolo, \& F. Bayron, Increased Static Postural Sway After Energy Drink Consumption a Randomized Trial, F1000Research, 6 (2017) 2036. [DOI]

[15] J.J. Trunzo, W. Samter, C. Morse, K. McClure, C. Kohn, J.E. Volkman, K. O'Brien, College Students' Use of Energy Drinks, Social Problem-Solving, and Academic Performance, Journal of Psychoactive Drugs, 46(5) (2014) 396-401. [DOI] | [PubMed]

[16] M.G. Rosario, L. Jamison, A. Hyder, The Influence of Energy Drinks on Lower Limb Neuromuscular Timing and Postural Sway in Healthy Young Adults, Journal of Public Health Issues and Practices, 4(1) (2020)163. [DOI]

[17] D. Moustakas, M. Mezzio, B.R. Rodriguez, M.A. Constable, M.E. Mulligan, E.B. Voura, Guarana Provides Additional Stimulation over Caffeine Alone in the Planarian Model, Plos One, 10(4) (2015). [DOI]

[18] A. Shumway-Cook, M.H. Woollacott, (2017) Motor control: Translating research into clinical practice, Lippincott Williams \& Wilkins, Philadelphia.

[19] I. Yunusa, I. Ahmad, Energy- Drinks: Composition and Health Benefits, Bayero Journal of Pure and Applied Sciences, 4(2) (2011) 186-191. [DOI] |
[20] J.P. Higgins, T.D. Tuttle, C.L. Higgins, Energy beverages: content and safety. Mayo Clinic Proceedings, 85(11) (2010) 1033-1041. [DOI] । [PubMed]

[21] T. Mottaghi, F. Khorvash, M. Maracy, N. Bellissimo, G. Askari, Effect of folic acid supplementation on nerve conduction velocity in diabetic polyneuropathy patients. Neurological Research, 41(4) (2019) 364-368. [DOI] । [PubMed]

\section{Funding}

This study was supported by the Texas Woman's University Faculty Small Grant Program awarded to the first author and PI.

\section{Ethics approval}

IRB approval TWU protocol \# 20107

\section{Authors Contribution}

All Authors contributed equally and approved the final version of this manuscript.

\section{Does this article screened for similarity?} Yes.

\section{Conflicts of Interest}

The authors have no conflicts of interest to declare that they are relevant to the content of this article.

\section{Informed consent}

Written consent was obtained from the participants

\section{About the License}

(C) The Author(s) 2021. The text of this article is open access and licensed under a Creative Commons Attribution 4.0 International License 